\title{
Increasing returns to scale and welfare: ranking the multiple deterministic equilibria
}

\section{Working Paper}

Author(s):

Bambi, Mauro; Saïdi, Aurélien

Publication date:

2008-10

Permanent link:

https://doi.org/10.3929/ethz-a-005678173

Rights / license:

In Copyright - Non-Commercial Use Permitted

Originally published in:

Economics Working Paper Series 08/99 


\title{
CER-ETH - Center of Economic Research at ETH Zurich
}

Increasing Returns to Scale and Welfare: Ranking the Multiple Deterministic Equilibria

\author{
Mauro Bambi and Aurélien Saïdi
}

Working Paper 08/99

October 2008

\section{Economics Working Paper Series}

\section{EIH}

Eidgenössische Technische Hochschule Zürich Swiss Federal Institute of Technology Zurich 


\title{
Increasing Returns to Scale and Welfare: Ranking the Multiple Deterministic Equilibria
}

\author{
Mauro Bambi \\ Center of Economic Research, ETH Zurich \\ and UCL, Université Catholique de Louvain \\ Aurélien Saïdi \\ Economix, Université Paris X - Nanterre
}

July 25, 2008

\begin{abstract}
We consider a real business cycle model with a productive externality and an aggregate nonconvex technology set $\grave{a} l a$ Benhabib and Farmer embodying capacity utilization, which exhibits indeterminacy of the steady state and multiplicity of deterministic equilibria under plausible values of the increasing returns to scale. The aim of the paper is to rank these different equilibria according to the initial value of consumption using both a linear-quadratic approximation, extensively explained by Benigno and Woodford [2006a, 2006b], and simulation methods. We study the implications of such a ranking in terms of smoothness of the welfare-maximizing trajectory and show that the welfaremaximizing consumption and labor paths are all the smoother since the level of increasing returns is low. At last, we show that this solution provides a good benchmark for judging the desirability of the stabilization policy proposed by Guo and Lansing [1997].

Keywords: Increasing returns, Local indeterminacy, Welfare analysis, Numerical Methods.

JEL classification: E32, E4, H61, O42, O47.
\end{abstract}

\section{Introduction}

In standard business cycle models, fluctuations in consumption and labor are generally welfare-diminishing compared to a smooth consumption/investment plan when the utility function is concave. In the presence of productive externalities, however, the welfare loss implied by fluctuations may be more than compensated by the gain inherited from the non-convexities of the production set. Christiano and Harrison [1999], for instance, have established that increasing volatility of labor may raise welfare in an economy with a non-convex technology set à la Benhabib and Farmer [1994]: for a given capital stock, by bunching hard work, agents are able to increase the average level of consumption without raising the average level of labor. When disutility of labor does not raise disproportionately to the additional utility procured by consumption during the periods of hard working, the "bunching" effect dominates the former negative "concavity" effect and makes the agents better-off. Thus, when the steady state equilibrium is locally indeterminate, that is when there is multiplicity of deterministic equilibria around the steady state, some equilibria may be welfare-improving compared to others. ${ }^{1}$

In the literature the possibility of stabilizing an economy characterized by local indeterminacy has been analyzed in such a framework by Guo and Lansing [1998] to prevent the agents from the endogenous fluctuations caused by indeterminacy and the opportunity of equilibrium path switching. ${ }^{2}$ Surprisingly, no much attention has been dedicated to the choice of the best equilibrium path on which the economy has to be stabilized, as if a stabilization scheme would necessarily improve welfare. But it is clear that a

\footnotetext{
${ }^{1}$ Contrary to Christiano and Harisson [1999] whose model relies on a welfare comparison between stochastic equilibria and the steady state, this paper focuses on a welfare comparison within the continuum of deterministic equilibria converging to the steady state in case of indeterminacy.

${ }^{2}$ Economic policies constructed to stabilize the economy by minimizing the variance of output have also been analyzed in models in which the level of externality required to get indeterminacy is less stringent than in the current framework. See for instance Guo and Harrison [2001] or Sim [2005].
} 
stabilizing policy can make the agents worse-off when expectations are pinned down on a suboptimal path. From Pareto's criterion viewpoint, any (decentralized) deterministic equilibrium path of the Benhabib and Farmer economy is not efficient as long as agents do not grasp the externality of production. Nevertheless, from a welfare viewpoint, these deterministic equilibria do not display the same level of utility: the optimization programme fails to determine which of them provides the maximum amount of welfare since they all satisfy the first order conditions and the transversality condition.

In this paper we make use of a linear-quadratic approximation to provide a welfare ranking of the different deterministic equilibria when the steady state is locally indeterminate. This method popularized by Benigno and Woodford [2006a, 2006b] consists in deriving a problem with linear constraints and a quadratic objective that approximates the exact problem. They show that more basic second-order perturbations can prove to be inaccurate in providing an exact welfare measure of alternative policy rules because they lead to a false approximation of the optimal policy rule. We thus rewrite the problem of the representative agent using this linear-quadratic approximation and determine the welfare-maximizing path that the original optimization program fails to select in the presence of sufficiently large increasing returns to scale. We prove that the starting value of consumption determines simultaneously the speed of the capital accumulation and the desirability of a deterministic path switching. Actually, we translate and investigate the concavity and the bunching effects developed by Christiano and Harrison in terms of monotonicity of the consumption/investment plan. It will be shown that the (decentralized) welfaremaximizing equilibrium displays a path all the less monotonic and an initial level of consumption all the higher since increasing returns to scale are high.

Bunching hard work in the very first periods makes capital accumulation faster. In the next periods, agents can benefit from the high level of capital stock by maintaining a high level of consumption but decreasing labor significantly. When increasing returns are high enough, reaching the welfare-maximizing capital stock requires relatively few time and effort, which explains the non-monotonicity of the equilibrium (or even the cyclicity of the solution) during the first periods. However, when increasing returns are close to the condition of indeterminacy, bunching hard work in the first periods is not sufficient to accumulate quickly a large capital stock, which would require huge levels of labor and a loss of welfare that next periods consumption cannot offset. Thus, when increasing returns to scale are not high enough, the welfare-maximizing equilibrium consists in minimizing the variance of labor, smoothing consumption and accumulating the capital stock progressively.

It has already been shown, for instance by Dupor and Lehnert [2002], that the derivation of the central planner optimal solution when indeterminacy occurs is not straightforward to compute, if not impossible formally. In this context, the welfare-maximizing path obtained by the way of the linear-quadratic approximation provides an excellent benchmark for judging the desirability of the stabilization policy proposed by Guo and Lansing. We prove that the tax policy they propose to prevent the economy from endogenous fluctuations generally deteriorates welfare compared to the welfare-maximizing equilibrium: the tax scheme smooths the consumption path while sufficient increasing returns to scale would require optimal fluctuations. We also provide an alternative policy able to pin down expectations on the welfaremaximizing equilibrium to reach the double-objection of stabilization and welfare-improvement. This can be done by targeting the rental rate on capital or the real wage at one or several periods of time.

After presenting briefly in section 2 the main characteristics of the model and the condition for indeterminacy, we assume this condition satisfied and define in section 3 the linear-quadratic approximation associated to the original problem and the dynamics of the economy. We also specify the set of monotonic consumption paths for any value of the parameters. These results are helpful in establishing the welfare ranking of section 4: simulation methods are used when a formal derivation appears too tricky. We compare the welfare-maximizing equilibrium path with the Guo-Lansing stabilization solution in section 5 and draw the conclusions for economic policy in section 6. Section 7 concludes this paper.

\section{Model Setup}

The general framework of this paper is a continuous-time model presented by Benhabib and Farmer model [1994] and modified by Wen [1998] in a discrete-time version embodying capacity utilization. This deterministic one-sector model with infinitely lived agents is characterized by a continuum of competitive 
firms, with the total number normalized to one. Social returns to scale are increasing since the presence of a factor-specific externality in production function. The individual firms use the following technology:

$$
\begin{aligned}
Y(t) & =A(t)[u(t) K(t)]^{a} L(t)^{b} \quad \text { with } 0<a<1 \text { and } a+b=1, \\
A(t) & =[\bar{u}(t) \bar{K}(t)]^{a \gamma} \bar{L}(t)^{b \gamma} \quad \text { with } \gamma>0,
\end{aligned}
$$

where $Y$ is the total output, , $u \in(0,1)$ capacity utilization, $\bar{K}$ and $\bar{L}$ represent the average economywide levels of capital $(K)$ and labor $(L)$. In equilibrium, $u=\bar{u}, K=\bar{K}$ and $L=\bar{L}$ and by making the parameters substitutions $\alpha \equiv a(1+\gamma)$ and $\beta \equiv b(1+\gamma)$, we get the aggregate production function:

$$
Y(t)=[u(t) K(t)]^{a(1+\gamma)} L(t)^{b(1+\gamma)} \equiv[u(t) K(t)]^{\alpha} L(t)^{\beta},
$$

which obviously exhibits increasing returns to scale since $\alpha+\beta>1$. In the same time, the economy is populated by a unit measure of identical infinitely lived consumers. The representative consumer, owner of capital, is endowed with one unit of time and maximizes:

$$
\max _{u, C, L} \int_{0}^{\infty}\left(\log C(t)-\frac{L(t)^{1+\chi}}{1+\chi}\right) e^{-\rho t} d t
$$

where $C$ and $L$ are the consumer's consumption and hours worked. Under the assumption that market factors are perfectly competitive, the budget constraint faced by the representative consumer is:

$$
\begin{aligned}
\dot{K}(t) & =(r(t)-\delta(t)) K(t)+w(t) L(t)-C(t), \\
\delta(t) & =\tau u(t)^{\theta} \quad \text { with } 0<\tau<1 \text { and } \theta>1,
\end{aligned}
$$

where $\chi \geq 0$ is the inverse of the Frisch elasticity of labor supply, $\rho>0$ is the discount rate and $\delta(t)>0$ the depreciation rate at time $t$. Consumers derive income by supplying capital and labor services to firms, taking the rate of return on capital $(r)$ and the real wage $(w)$ as given.

Following Wen [1998], $\theta$ is chosen strictly greater than one in order to guarantee an interior steady state value of the capacity utilization. Capacity utilization amplifies and propagates business cycle: capital is more intensively used during economic booms when its marginal product is high.

In a symmetric perfect foresight equilibrium in which consumers maximize utility and firms make zero profit, the equilibrium conditions can be expressed as an autonomous pair of difference equations after deriving the first-order conditions of the maximization problem (see appendix for more details):

$$
\begin{aligned}
\dot{k} & =\left(1-\frac{a}{\theta}\right) e^{\mu_{0}+\mu_{1} k+\mu_{2} c}-e^{c-k} \\
\dot{c} & =a\left(1-\frac{1}{\theta}\right) e^{\mu_{0}+\mu_{1} k+\mu_{2} c}-\rho
\end{aligned}
$$

where $x=\ln X, \mu_{0}=-\frac{\alpha(1+\chi) \ln (a / \theta \tau)+\theta \beta \ln b}{\theta \beta+\alpha(1+\chi)-\theta(1+\chi)}, \mu_{1}=\frac{\theta[(1-\alpha)(1+\chi)-\beta]}{\theta \beta+\alpha(1+\chi)-\theta(1+\chi)}$ and $\mu_{2}=\frac{\theta \beta}{\theta \beta+\alpha(1+\chi)-\theta(1+\chi)}$.

It is straightforward to show that the above dynamical system possesses a unique interior steady state and that under the condition $\beta \frac{\theta}{\theta-\alpha}-1-\chi>0$ this steady state is indeterminate. ${ }^{3}$ In the neighborhood of this steady state, there exists a continuum of equilibrium paths converging to it. In this framework, the rational expectations hypothesis, which usually lead to a unique equilibrium path, cannot discriminate between the different equilibrium paths. However from a welfare viewpoint these paths are not equivalent and a welfare ranking can be established.

\footnotetext{
${ }^{3}$ This condition for indeterminacy is necessary and sufficient in our continuous-time setup; however, in the discrete-time model of Wen [1998], it is only necessary. For a perfectly elastic labor supply, that is when $\chi=0$ and for $\theta$ arbitrarily closed to 1 , the condition for indeterminacy collapses to $\alpha+\beta>1$ and is always satisfied under the assumption of increasing returns to scale.
} 


\section{Solving the model}

\subsection{Linear-Quadratic approximation}

In this section, we make use of the method developed by Fleming [1971], applied by Magill [1977] and recently revisited by Benigno and Woodford [2006a, 2006b] that substitutes to the maximand (1) a quadratic objective and to the law of motion of capital, equation (2), a linear constraint. This approximation does not consist of a basic Taylor expansion of the utility function but requires to substitute to the linear terms of the Taylor expansion terms of order 2 obtained by a second-order approximation of the first-order conditions. ${ }^{4}$

By contrast with a basic Taylor expansion, it can be proved that the linear-quadratic (LQ) approximation leads to a correct linear approximation of the optimal policy rule. It is thus equivalent to use this method to derive the linear approximations of capital and consumption around the steady state or the original problem. Furthermore, it is now well known that first-order approximation techniques are not always well suited to handle welfare comparisons. ${ }^{5}$ The LQ approximation, whose objective function is quadratic, suits perfectly for problems of welfare comparisons.

From now, it will be assumed that the model exhibits local indeterminacy: both eigenvalues have strictly negative real part and all equilibrium paths converge to the same steady state. Maximizing (1) is then equivalent to maximizing:

$$
\max _{u, C, L} \int_{0}^{\infty} \tilde{U}(c(t), l(t)) e^{-\rho t} d t
$$

with:

$$
\begin{aligned}
\tilde{U}(c, k) & \equiv c(t)-\frac{e^{l(t)(1-\chi)}}{1-\chi}-c_{s}+\frac{e^{l_{s}(1-\chi)}}{1-\chi} \\
& =[c, l(k, c)] D^{2} U(c, l)[c, l(k, c)]^{\prime}+[c, l(k, c), k] D^{2} \dot{k}(c, l, k)[c, l(k, c), k]^{\prime} \\
& =\underbrace{-(1-\chi) e^{(1-\chi) \bar{l}} \tilde{l}(k, c)^{2}}_{U_{1}(t)}+\underbrace{e^{-\bar{c}}\left[\Psi\left(\mu_{1}^{\prime} \tilde{k}+\mu_{2}^{\prime} \tilde{c}\right)^{2}-(\Psi-\delta)(\tilde{c}-\tilde{k})^{2}\right]}_{U_{2}(t)}+\mathcal{O}\left(\|(c, k)\|^{3}\right) \\
& \equiv \hat{U}(k, c)+\mathcal{O}\left(\|(c, k)\|^{3}\right),
\end{aligned}
$$

where $c_{s}$ and $l_{s}$ are the steady state log-values of consumption and labor, $\tilde{l}(k, c)=\frac{\tilde{c}-\alpha \frac{\theta-1}{\theta-\alpha} \tilde{k}}{\beta \frac{\theta}{\theta-\alpha}-1-\chi}$ for $\beta \frac{\theta}{\theta-\alpha}-$ $1-\chi \neq 0, \mu_{1}^{\prime}=-\frac{\theta-1}{\theta-\alpha} \frac{a(1+\chi)}{\beta \frac{\theta}{\theta-\alpha}-1-\chi}-1$ and $\mu_{2}^{\prime}=\frac{\theta b}{(\theta-\alpha)\left(\beta \frac{\theta}{\theta-\alpha}-1-\chi\right)}, D^{2} U$ and $D^{2} \dot{k}$ are the Hessian matrix of the utility function $U$ and $\dot{k}$ (when the agents do not internalize the productive externality).

The quadratic approximation of $\tilde{U}(c(t), l(t))$, namely $\hat{U}(c(t), l(t))$, associated to the linear approximation of the law of motion of capital, equation (2), form the LQ approximation of the original problem.

The first-order conditions of the reformulated programme give the approximations of capital and consumption around the steady state, expressed in terms of deviation of the two variables $k(t)$ and $c(t)$ from their steady state values $k_{s}$ and $c_{s}$, i.e. $\tilde{x}(t)=\ln X(t)-\ln X_{s}$. We get:

$$
\left[\begin{array}{l}
\tilde{k}(t) \\
\tilde{c}(t)
\end{array}\right] \simeq\left[\begin{array}{ll}
\eta_{1} v_{11} & \eta_{2} v_{12} \\
\eta_{1} v_{21} & \eta_{2} v_{22}
\end{array}\right]\left[\begin{array}{l}
e^{\lambda_{1} t} \\
e^{\lambda_{2} t}
\end{array}\right]
$$

with

$$
V=\left[\xi_{1}: \xi_{2}\right]=\left[\begin{array}{ll}
v_{11} & v_{12} \\
v_{21} & v_{22}
\end{array}\right]=\left[\begin{array}{cc}
{\left[1-\mu_{2}\left(1-\frac{a}{\theta}\right)\right] \Psi-\delta} & {\left[1-\mu_{2}\left(1-\frac{a}{\theta}\right)\right] \Psi-\delta} \\
{\left[1+\mu_{1}\left(1-\frac{a}{\theta}\right)\right] \Psi-\delta-\lambda_{1}} & {\left[1+\mu_{1}\left(1-\frac{a}{\theta}\right)\right] \Psi-\delta-\lambda_{2}}
\end{array}\right],
$$

where $\Psi \equiv(\rho+\delta) / a, \delta=\frac{\rho}{\theta-1}$ is the steady state value of capital depreciation and $\xi_{1}$ and $\xi_{2}$ are eigenvectors associated to the eigenvalues $\lambda_{1}$ and $\lambda_{2}$, which can be obtained after computing the Jacobian of the system

\footnotetext{
${ }^{4}$ See Benigno and Woodford [2006a, 2006b] for more details in a discrete-time framework.

${ }^{5}$ This is especially the case for stochastic models, as already shown by Kim and Kim [2003] or Schmitt-Grohe and Uribe [2004] among others, but the conclusion remains valid for deterministic framework as ours.
} 
formed by equations (3) and (4). Moreover, given a starting point $[K(0), C(0)]$, we apply Cramer's rule and deduce:

$$
\begin{aligned}
\eta_{1} & =\frac{v_{22} \tilde{k}(0)-v_{12} \tilde{c}(0)}{v_{11} v_{22}-v_{12} v_{21}} \\
\eta_{2} & =\frac{v_{11} \tilde{c}(0)-v_{21} \tilde{k}(0)}{v_{11} v_{22}-v_{12} v_{21}}
\end{aligned}
$$

\subsection{Monotonic paths}

To understand the economic implications of the equilibrium paths ranking in terms of intertemporal consumption smoothness, we study in this subsection the conditions on $c(0)$ under which the path is monotonic. It is reminded that monotonicity only occurs when eigenvalues are real. In the following, we will assume without loss of generality that $\lambda_{1}<\lambda_{2}<0$.

Define the stable arms of the dynamics the two equilibrium paths:

$$
\tilde{c}(t)=\eta_{i} v_{2 i} e^{\lambda_{i} t}, \quad i=\{1,2\}
$$

and let $c_{0, \xi_{i}}, i=\{1,2\}$, be the starting log-values of consumption of such a path for a given initial stock of capita $K(0)$. These values are such that respectively $\eta_{1}$ and $\eta_{2}$ equalize zero, that is:

$$
\begin{aligned}
& c_{0, \xi_{1}}=c_{s}+\tilde{k}(0) \frac{v_{21}}{v_{11}} \\
& c_{0, \xi_{2}}=c_{s}+\tilde{k}(0) \frac{v_{22}}{v_{12}} .
\end{aligned}
$$

The following proposition holds:

Proposition 1 For a given initial stock of capital $K(0)<K_{s}$ (resp. $K(0)>K_{s}$ ), there exists a strictly positive (resp. negative) $\varepsilon^{*}$ such that for $c(0) \in\left[c_{0, \xi_{2}}-\varepsilon^{*}, c_{0, \xi_{1}}\right]$ (resp. $\left[c_{0, \xi_{1}}, c_{0, \xi_{2}}-\varepsilon^{*}\right]$ ) equilibrium paths of consumption are monotonic.

Proof. See Appendix A.3.

\section{Welfare comparisons}

Once the local dynamics of the economy has been characterized through the LQ approximation, the next objective is to select the initial level of consumption which the highest level of welfare is associated to. It is worth noting here that a local analysis means as usual a choice of initial conditions for the aggregate variables sufficiently close to their steady state values. In particular, we have referred to the contributions of Russell and Zecevic [1998, 2000] in order to select initial level of consumptions in the region of stability. We thus proceed in two steps.

First, we restrict the analysis to the lower bound of the externality above which indeterminacy occurs, that is when $\beta \frac{\theta}{\theta-\alpha}$ tends to $1-\chi$. We thus determine analytically the unique equilibrium path and the related welfare-maximizing starting value of consumption. ${ }^{6}$ The linear-quadratic approximation of the utility function cannot be used for this specific case since the Jacobian matrix is not properly defined. ${ }^{7}$ However, some conjectures can be drawn for higher levels of the increasing returns when indeterminacy occurs.

Second, making use of the linear-quadratic approximation and simulation methods we enlarge the range of increasing returns to scale: we are then able to determine precisely a good approximation of the true value of the welfare-maximizing starting value of consumption and the behavior of the related consumption/investment plan whatever the values of the parameters.

\footnotetext{
${ }^{6}$ By the vocable "welfare-maximizing path" we mean the path making the agents better-off from a decentralized viewpoint, that is when the productive externality is considered as given. From the social planner viewpoint, that is when the agents take into account the impact of their decisions on the utility and the income of the others, this path is undoubtedly suboptimal. However, within the equilibrium paths selected by the representative agent the welfare-maximizing path returns the higher level of utility. It is then important not to liken the optimal path of the economy to the welfare-maximizing path.

${ }^{7}$ More precisely, the competitive equilibrium path of the main aggregate variables can be directly derived without linearizing the system.
} 


\subsection{Specific case for $\beta \frac{\theta}{\theta-\alpha}=1+\chi$}

When $\beta \frac{\theta}{\theta-\alpha}$ tends to $1+\chi$, neither the approximation of $\tilde{l}$ nor the Jacobian matrix are defined (some terms tend to infinity). For this specific value of the increasing returns, the equilibrium path is unique and the following proposition holds:

Proposition 2 When $\beta \frac{\theta}{\theta-\alpha}=1+\chi$, the welfare-maximizing path is monotonic and starts with $c(0)=$ $c_{0, \xi_{2}}$.

Proof. When $\beta \frac{\theta}{\theta-\alpha}$ tends to $1+\chi$, equations (15) and (16) collapse to $c(t)=\alpha \frac{\theta-1}{\theta-\alpha} k(t)+\ln b+\frac{\alpha}{\theta-\alpha} \ln \frac{a}{\theta \tau}$, or equivalently: $\tilde{c}=\alpha \frac{\theta-1}{\theta-\alpha} \tilde{k}$. By equalizing $\dot{C} / C$ and $\alpha \frac{\theta-1}{\theta-\alpha} \dot{K} / K$ and using the first-order condition respect to $u$ :

$$
u=\left[\frac{a}{\tau \theta} K^{\alpha-1} L^{\beta}\right]^{\frac{1}{\theta-\alpha}},
$$

the welfare-maximizing relationship between $K$ and $L$ can be found:

$$
L=\left[b \varphi-\rho \varphi\left(\frac{a}{\tau \theta}\right)^{-\frac{\alpha}{\theta-\alpha}} K^{-\frac{\theta(1-\alpha)}{\theta-\alpha}}\right]^{\frac{\theta-\alpha}{\theta \beta}},
$$

where $\varphi^{-1} \equiv\left(1-\frac{a}{\theta}\right)\left(\alpha \frac{\theta-1}{\theta-\alpha}-a\right)$.

There is a correspondence between $K, C, L$ and $u$ : the welfare-maximizing path is then unique.

By substituting the welfare-maximizing values of $u, L$ and $C$ respect to $K$ in $\dot{K}$, one can show that for $K<K_{s}$ (resp. $\left.K>K_{s}\right) \dot{K}>0$ (resp. $\dot{K}<0$ ) and then that the capital stock evolves monotonically to the steady state. So do capacity utilization, labor and consumption.

For a given $K_{0}$ the welfare-maximizing path is such that $\tilde{c}(0)=\alpha \frac{\theta-1}{\theta-\alpha} \tilde{k}(0)$. Since $v_{22} / v_{12}$ tends to $\frac{\theta-1}{\theta-\alpha}$ when $\beta \frac{\theta}{\theta-\alpha}$ tends to $1+\chi$, according to equation (8) the optimal path starts with an initial level of consumption $c(0)=c_{0, \xi_{2}}$.

By continuity of the first-order conditions respect to $\gamma$ :

$$
C L^{-\chi}=K^{a(1+\gamma)} L^{(1-a)(1+\gamma)-1},
$$

and

$$
u=\left[\frac{a}{\tau \theta} K^{a(1+\gamma)-1} L^{b(1+\gamma)}\right]^{\frac{1}{\theta-a(1+\gamma)}},
$$

the relationship between $\tilde{c}(t)$ and $\tilde{k}(t)$ is (approximatively) maintained also when $\gamma$ increases and $\beta \frac{\theta}{\theta-\alpha}=$ $1+\chi>0$ (however close enough to zero). Since $\tilde{c}(t) \sim \alpha \tilde{k}(t)$ then

$$
c(0) \sim c_{0, \xi_{2}} .
$$

Some conclusions about the welfare-maximizing path can be drawn as $\beta \frac{\theta}{\theta-\alpha}$ increases from $1+\chi$ : as already seen, $U_{1}(t)$ is always negative whereas for $\tilde{c}(t) \sim \alpha \tilde{k}(t), U_{2}(t) \sim \delta e^{-\bar{c}}\left(1-\frac{\theta-a}{\theta a}\right)^{2} c(t)^{2}$ and is maximum for $|c(0)|$ as high as possible, i.e. $c(0)=c_{0, \xi_{2}}-\varepsilon^{*}{ }^{8}$. When $\beta \frac{\theta}{\theta-\alpha}-1-\chi$ increases from zero, increasing $|\tilde{l}(t)|$ yields a mid decrease of $U_{1}(t)$ for given $\tilde{c}$ and $\tilde{k}$ while $U_{2}(t)$ can be increased provided $c(0)$ gets closer to $c_{0, \xi_{2}}-\varepsilon^{*}$.

Thus, as $\beta \frac{\theta}{\theta-\alpha}$ increases from $1+\chi$, we expect a decrease of the welfare-maximizing $c(0)$ relatively to $c_{0, \xi_{2}}$. We then switch to simulation methods to enlarge the set of possible levels of increasing returns to scale and check whether or not the agents have interest to keep choosing a non-monotonic equilibrium path, i.e. a starting value of consumption that is lower than $c_{0, \xi_{2}}-\varepsilon^{*}$ or higher than $c_{0, \xi_{1}}$. This is the objective of the next subsection.

\footnotetext{
${ }^{8}$ Since $\tilde{c}(t) \sim \alpha \tilde{k}(t)$, the welfare-maximizing path must be monotonic and $c(0)$ must be greater or equal to $c_{0, \xi_{2}}-\varepsilon^{*}$.
} 


\subsection{Simulation methods}

For a predetermined stock of capital $K(0)$ and a given initial level of consumption $C(0)$, we can compute using system (6) the multiple equilibrium paths of capital and consumption each of them associated to a different choice of initial consumption. Once the associated competitive equilibrium path of labor has been derived using the first-order conditions (15) and (16):

$$
\left(\beta \frac{\theta}{\theta-\alpha}-1-\chi\right) \tilde{l}(t)=\tilde{c}(t)-\alpha \frac{\theta-1}{\theta-\alpha} \tilde{k}(t)
$$

and the log-variations of consumption and labor have been replaced in the objective function (5), the approximated maximization problem can be reformulated as follows:

$$
\max _{c(0)} \int_{0}^{\infty} \hat{U}(c(0)) e^{-\rho t} d t .
$$

Computationally, we solve numerically the integral and obtain a quadratic approximation of the maximand (11) of the form:

$$
\hat{U}[c(0)]=n_{1} c(0)^{2}+n_{2} c(0)+n_{3},
$$

where $n_{1}<0$ and whose maximum is reached for: ${ }^{9}$

$$
c^{*}(0)=-2 n_{1} / n_{2}
$$

We parameterize the economy as follows. The capital share fits the average annual value of $a=0.29$ over the 1950-85 period as reported in Greenwood et al. [1988]. Following Hansen [1985] and the argument that labor, if indivisible at the individual level, behaves as if it were infinitely elastic, the inverse of the Frisch elasticity of labor is set to $\chi=0$. The values of $\tau$ and $\theta$ are assigned to match both the long-run average of $81 \%$ for the past several years found by the Federal Reserve Bank [2008] and the depreciation rate of capital of $\delta=0.12$ estimated by Nadiri and Prucha [1997]. The discount rate $\rho=0.02$ corresponds approximatively to a discount factor of 0.98 (which usually oscillates between 0.95 and 0.99 in the literature). The initial value of capital is set close enough (but not too close) to the steady state at $k_{0}=0.9 k_{s} .{ }^{10}$ Then, for different sizes of the increasing returns to scale, $\gamma$, we obtain figures 1 and 2 .

On figure 1, we have sketched the welfare-maximizing value of $c(0)$ in addition to $c_{0, \xi_{2}}-\varepsilon^{*}$ and $c_{0, \xi_{2}}$ as the degree of increasing returns to scale $\gamma$ increases for values satisfying the condition for indeterminacy. These results confirm proposition 2, which predicts that within the set of monotonic paths, the maximizing welfare equilibrium starts with an initial level of consumption $c(0)=c_{0, \xi_{2}}$. They also confirm our conjecture that this initial level tends to get closer to $c_{0, \xi_{2}}-\varepsilon^{*}$ as $\gamma$ increases.

When the real part of both eigenvalues merge (as shown on figure 2) and become complex, it can be easily seen from equations $(7)$ and (8) that $\operatorname{Re}\left(v_{21} / v_{11}\right)=\operatorname{Re}\left(v_{22} / v_{12}\right)$ and then that $\operatorname{Re}\left(c_{0, \xi_{1}}\right)=$ $\operatorname{Re}\left(c_{0, \xi_{2}}\right)=\operatorname{Re}\left(c_{0, \xi_{2}}-\varepsilon^{*}\right)$. For higher level of increasing returns, the welfare-maximizing path is no longer monotonic. However, it must be noticed that the welfare-maximizing path becomes non-monotonic before this threshold. Since $c_{0, \xi_{2}}-\varepsilon^{*}$ increases faster than $c^{*}(0)$ there is a level of increasing returns above which the maximum welfare is reached for an initial level of consumption $c^{*}(0)$ outside the range $\left[c_{0, \xi_{2}}-\varepsilon^{*}, c_{0, \xi_{1}}\right]$, meaning that the welfare-maximizing path is fluctuating. Smoothness of the consumption path shrinks and definitely disappears as the eigenvalues become complex: the welfare-maximizing path is then cycling. The presence of cycles is not surprising: Dupor and Lehnert [2002] have already investigated for specific values of the increasing returns and labor elasticity the social planner's solution of such an economy and evoked the plausibility of optimal cyclicity. However, in the absence of precise formulation of this optimal path the welfare-maximizing path we found remains the best benchmark for any stabilization policy.

\footnotetext{
${ }^{9}$ For given levels of labor elasticity and capital share, large degrees of increasing returns change the physiognomy of $\hat{U}[c(0)]: n_{1}>0$. There is a shift in the welfare-maximizing starting value of consumption and a corner solution appears. However, these cases are not easily tractable since we do not have an exact measure of the range of plausible values for the starting value of consumption.

${ }^{10}$ Alternative computations have been derived with $k_{0}=0.99 k_{s}$ and $k_{0}=0.999 k_{s}$ but do not affect the conclusions. The difference in welfare from one path to another is however smaller.
} 
Figure 1:

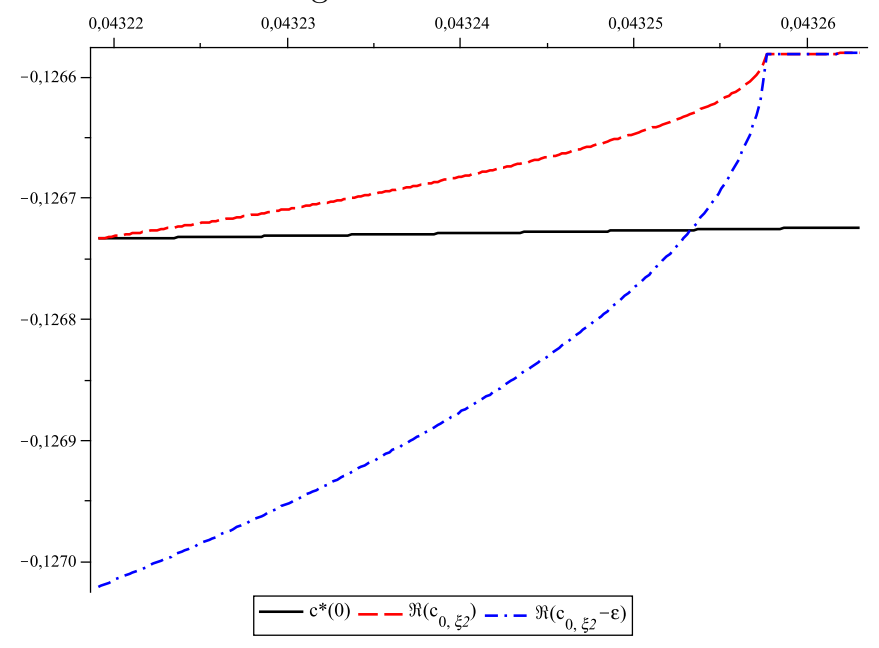

Figure 2:

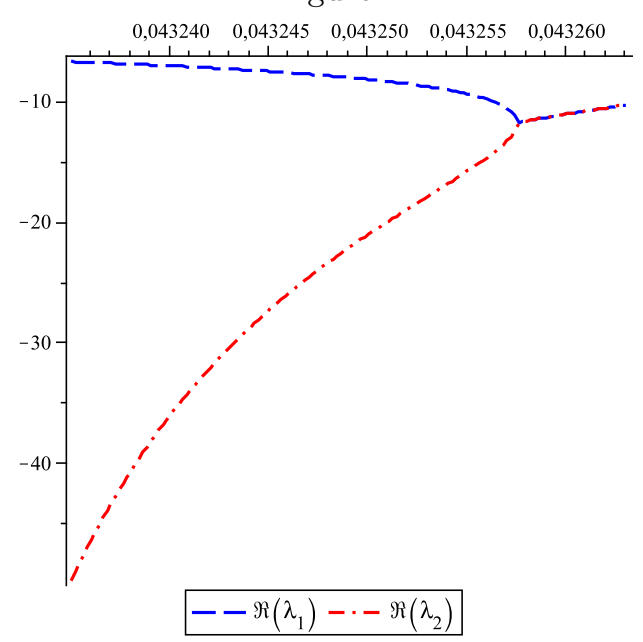

A sensitivity analysis shows that the configuration above is stable for a change in the parameter values. However, a decrease in labor elasticity (or equivalently an increase of $\chi$ ) strengthens the condition for indeterminacy and imposes a shift to the right of the point of intersection between the dashed line and the dashed-dotted line. By raising the value of $\theta$, a decrease of the depreciation rate $\delta$ or an increase of the rate of time preference $\rho$ have the same impact. ${ }^{11}$

A point has to be stressed however: it is clear from the simulations below that the point of intersection (PI) is all the higher compared to the lower bound of the increasing returns to scale (IRS) required to get indeterminacy since the condition for indeterminacy is strengthened.

\begin{tabular}{||c|c|c||}
\hline \hline Parameter values & Lower bound of IRS & \%-deviation of the PI \\
\hline \hline$\delta=.12, \rho=.02, \chi=0$ & 0.043 & $0.081 \%$ \\
\hline$\delta=.10, \rho=.02, \chi=0$ & 0.051 & $0.122 \%$ \\
\hline$\delta=.10, \rho=.05, \chi=0$ & 0.107 & $0.457 \%$ \\
\hline$\delta=.10, \rho=.05, \chi=0.25$ & 0.313 & $2.078 \%$ \\
\hline \hline
\end{tabular}

Thus, the range of monotonic paths enlarges as $\delta, \rho$ or $\chi$ increases. As a consequence, the more the condition for indeterminacy is strengthened the more likely the welfare-maximizing path is to be monotonic. The one-sector Benhabib and Farmer model [1994] provides a limit case (figures 3 and 3): for low elasticities of labor and high capital shares, eigenvalues may not merge, the welfare-maximizing values of initial consumption may remain inside the range $\left[c_{0, \xi_{2}}-\varepsilon^{*}, c_{0, \xi_{2}}\right]$ and non-monotonic paths may never be welfare-maximizing.

At last, it is worth noting that the welfare-maximizing path is not always increasing. For some values of the parameters, the steady state value of consumption is decreasing as the degree of increasing returns increases. These cases correspond to mathematical oddness and are irrelevant for the purpose of this paper: since the labor steady state value is strictly lower than unity, it can appear that rising the degree of the increasing returns have a negative impact on aggregate production. When there is a positive relationship between increasing returns and production (that is for a positive externality of production), the welfare-maximizing path is unambiguously increasing.

In any case, whether decreasing or not, the welfare-maximizing path exhibits a decreasing ratio $C^{*}(0) / C_{s}$ as the degree of increasing returns rises.

\section{Economic arguments}

In optimal growth models à la Benhabib and Farmer with social increasing returns to scale and productive externalities, Christiano and Harrison [1999] distinguish two effects affecting the consumption/investment

\footnotetext{
${ }^{11}$ The impact of $a$ on the condition for indeterminacy is more ambiguous: an increase strengthens the condition for $1+\gamma-\theta>0$ and weakens it otherwise.
} 
Figure 3:

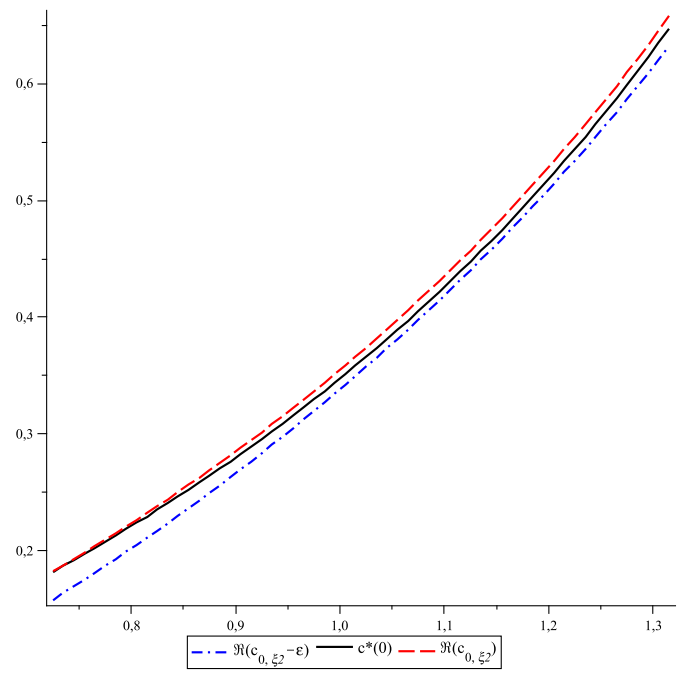

Figure 4:

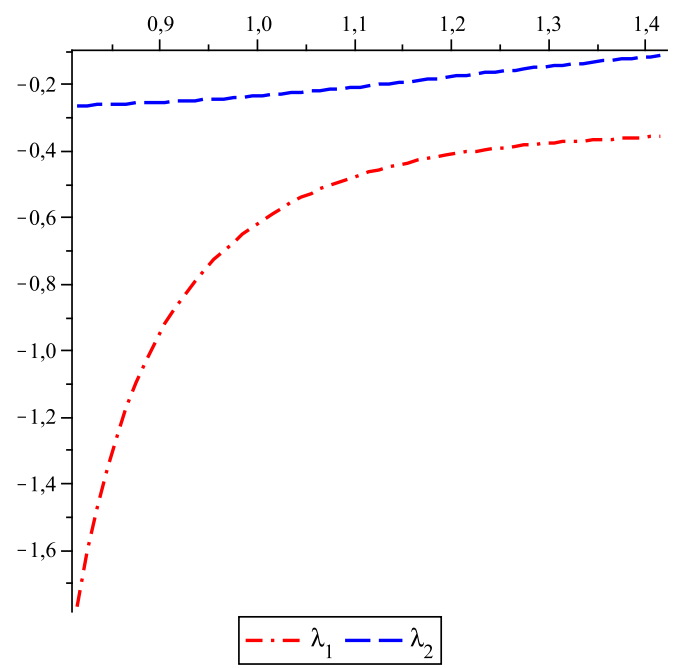

plans. For a given technological coefficient (a given productive externality), the concavity of the utility function prevents from fluctuations which deteriorate welfare. This "concavity effect" leads to choose monotonic equilibria and smooth consumption and labor over time so as to maximize the representative agent's welfare. However, as the productive externality increases with the average economy-wide levels of capital and labor, it may be welfare-improving to bunch hard work in the first periods to boost capital accumulation in order to benefit from a higher productive externality in the next while maintaining a relatively lower level of labor.

Figure 5:

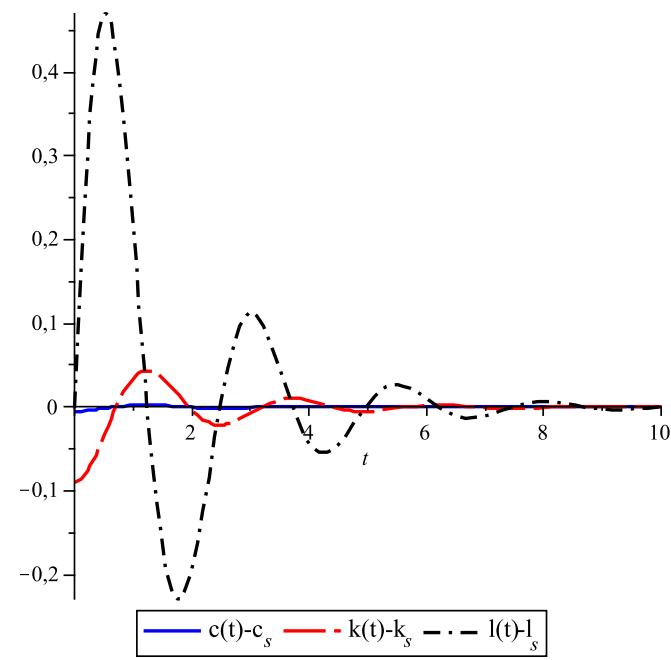

Figure 6:

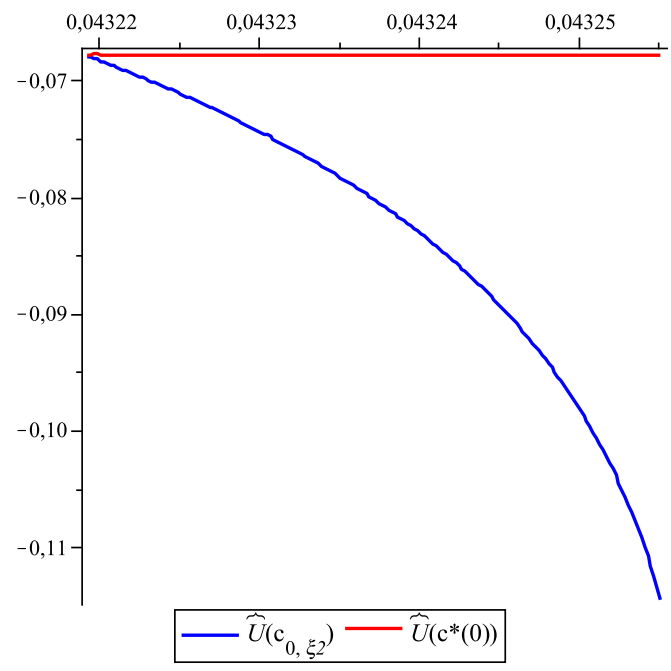

When the "bunching effect" dominates the "concavity effect", agents bring forward a part of their labor supply, raising the average level of consumption and decreasing labor after a while. On the figure above, we pictured the welfare-maximizing paths of capital, consumption and labor for $\gamma=0.044$ : they clearly show how cycles can make the agents better-off.

On the one hand, a higher level of increasing returns to scale makes capital accumulation larger for the same amount of worked hours or equivalently allows the representative agent to raise consumption without raising labor. On the other hand, the higher the level of increasing returns to scale, the higher the steady state value of capital: the average level of labor required to reach this steady state value appears to be higher when the consumption path is monotonic since agents do not benefit from the nonconvexities of the production function. The conjunction of these two effects explain why it is important 
to bunch hard work when the degree of increasing returns to scale is sufficient. In that case, consumption and capital remain during several periods above their steady state values whereas labor remains below. Agents accumulate the capital stock during the first periods, which erodes gradually afterwards: they use this capital stock accumulated to conciliate a high level of consumption with a moderate level of labor.

However, even though it can be conjectured from Dupor and Lehnert [2002] that an optimality of cycles is intrinsically associated to indeterminacy provided there are aggregate decreasing returns on capital, the welfare-maximizing path may be monotonic: capital, consumption and labor lay below their steady state values forever (but get closer over time). ${ }^{12}$ Since firms consider the productive externality as given while at the symmetric equilibrium it increases when firms increase their production, there exists a threshold below which it is not optimal to bunch hard work if not intensively. For low level of increasing returns to scale, cycles might be optimal provided agents sufficiently bunch hard work: if not, the capital stock does not increase sufficiently while disutility of labor significantly rises. Agents are not able to raise the average level of consumption sufficiently to compensate the welfare loss caused by the periods of hard working. As the degree of increasing returns increases the decentralized non-monotonic equilibrium paths provide periods of hard working more likely to sufficiently rise the capital stock and finally the average level of consumption that compensate the welfare loss of the periods of hard working.

Thus, when the degree of increasing returns to scale is low, choosing a monotonic path allows the agents to gradually accumulate the capital stock and prevents them from paying a stringent tribute in terms of welfare that the increase in consumption cannot compensate. This is especially the case when the level of increasing returns is close to the lower bound insuring indeterminacy: the "bunching effect" is not strong enough (as it might be from a social planner's viewpoint) to dominate the "concavity effect". Far from accelerating capital accumulation, agents are better-off when they smooth consumption and labor over time. As increasing returns become more and more important, the "bunching effect" is more likely to offset the "concavity effect" and the welfare-maximizing path is all the less monotonic since the degree of externalities increases.

Whether monotonic or not, the welfare-maximizing path embodies a decreasing $C^{*}(0) / C_{s}$ ratio as the degree of increasing returns raises. This decrease means that the welfare-maximizing initial value of consumption increases slower than its steady state value. Actually, as the degree of increasing returns raises, agents can improve their welfare by both increasing the average level of consumption (then its steady state value) and decreasing or increasing moderately the number of worked hours, especially during the first periods: the rise in the productive externality alone insures a faster and higher accumulation of the capital stock. As pictured in figure 6, although the welfare-maximizing difference $\hat{U}\left(c^{*}(0)\right)$ increases very slightly as the degree of increasing returns raises, choosing the minimizing labor variance path starting with $c(0)=c_{0, \xi_{2}}$ deteriorates exponentially welfare. The loss of welfare remains limited as long as the welfare-maximizing path is monotonic; as soon as the welfare-maximizing path exhibits cycles, the loss substantially increases and reveals the price to pay for not using the bunching effect to reach continuously increasing steady state values of consumption and capital.

Finally, since the welfare-maximizing path is much more easier to compute than the optimal equilibrium of this economy, it represents a second-best equilibrium and an important observation for judging the desirability of a stabilization policy, whose performances in terms of welfare can be compared with it.

\section{Stabilization policy and welfare-maximizing path}

\subsection{Guo and Lansing tax policy}

We apply to the Wen framework [1998] a specific version of the Guo and Lansing stabilization policy developed in the one-sector Benhabib and Farmer model [1994]. This policy consists of a countercyclical

\footnotetext{
${ }^{12}$ It can be proved that for optimal values of the capital stock and its costate variable the optimal value of labor must be a corner solution when the condition for indeterminacy holds in Benhabib and Farmer [1994], Benhabib and Farmer [1996] or Wen [1996]. The existence of a chattering solution in such model is very likely. By contrast, Christiano and Harrison [1999] have shown that the solution is monotonic when the aggregate production function exhibits constant marginal returns on capital, that is when $\alpha=1$.
} 
tax on production $\tau(t)$ (or equivalently a tax on both capital and labor incomes): ${ }^{13}$

$$
\tau(t)=1-\left(\frac{Y_{s}}{\bar{Y}(t)}\right)^{\phi} \quad \text { with } \quad \phi>0 .
$$

In this case, each agent faces the same tax rate which increases with the national income. Finally, the law of motion of capital becomes:

$$
\dot{K}(t)=[(1-\tau(t)) r(t)-\delta] K(t)+(1-\tau(t)) w(t) L(t)-C(t)+T(t) .
$$

It can be shown that the steady state does not vary from what we have previously computed while the new eigenvectors are such that:

$$
V=\left[\xi_{1}: \xi_{2}\right]=\left[\begin{array}{ll}
v_{11} & v_{12} \\
v_{21} & v_{22}
\end{array}\right]=\left[\begin{array}{cc}
{\left[1-\mu_{2}^{\prime}\left(1-\frac{a}{\theta}\right)\right] \Psi-\delta} & {\left[1-\mu_{2}^{\prime}\left(1-\frac{a}{\theta}\right)\right] \Psi-\delta} \\
{\left[1+\mu_{1}^{\prime}\left(1-\frac{a}{\theta}\right)\right] \Psi-\delta-\lambda_{1}^{\prime}} & {\left[1+\mu_{1}^{\prime}\left(1-\frac{a}{\theta}\right)\right] \Psi-\delta-\lambda_{2}^{\prime}}
\end{array}\right],
$$

where:

$$
\begin{aligned}
\mu_{1}^{\prime} & =\frac{\theta[(1-\alpha(1-\phi))(1+\chi)-\beta(1-\phi)]}{\theta \beta(1-\phi)+\alpha(1-\phi)(1+\chi)-\theta(1+\chi)} \\
\mu_{2}^{\prime} & =\frac{\theta \beta(1-\phi)}{\theta \beta(1-\phi)+\alpha(1-\phi)(1+\chi)-\theta(1+\chi)} .
\end{aligned}
$$

The modified condition for determinacy is satisfied when $\beta \frac{\theta}{\theta /(1-\phi)-\alpha}-1-\chi<0 .{ }^{14}$ For the minimum value of $\phi$ insuring determinacy, the stabilizing saddle-path solution so obtained is such that:

$$
\begin{gathered}
\tilde{c}(t) \sim \tilde{c}_{0, \xi_{2}} e^{\lambda_{2}^{\prime}} \\
\tilde{k}(t) \sim \tilde{k}(0) e^{\lambda_{2}^{\prime}},
\end{gathered}
$$

with:

$$
\lambda_{2}^{\prime}=a(\Psi-\delta) \frac{1-\alpha(1-\phi)}{a-\alpha(1-\phi)} .
$$

The stabilizing saddle-path solution is then close to the equilibrium path starting with $c(0)=c_{0, \xi_{2}}$ : both paths start with the same initial conditions $[k(0), c(0)]$ and converge to the same steady state. Furthermore, their growth rates, respectively $\lambda_{2}^{\prime}$ and $\lambda_{2}$ are approximatively similar for $\phi$ close enough to zero (see appendix A.4 for more details). In the limit case, when $\frac{\theta}{\theta-\alpha}$ tends to $1+\chi$ an infinitely small $\phi$ can be chosen to suppress indeterminacy and the stabilizing saddle-path equilibrium tends to merge with the indeterminate equilibrium path starting with $c(0)=c_{0, \xi_{2}}$. In that case, we have shown that this latter path coincides with the welfare-maximizing path. The Guo and Lansing policy is then as good as the welfare-maximizing path for infinitely low levels of increasing returns.

However, it is clear on the simulations above that the loss in welfare for an agent maintaining $c_{0, \xi_{2}}$ as a starting level of consumption (which is the case of the Guo and Lansing policy) increases exponentially with the level of increasing returns. As this level goes up the "bunching effect" raises and theoretically may offset the "concavity effect": the welfare-maximizing path is less and less monotonic while the Guo and Lansing tax policy leads the agents to smooth their consumption/investment plans. For plausible values of the increasing returns, the cost of the stabilization policy appears rapidly prohibitive and it seems more interesting to find an alternative stabilization policy that pins down expectations on the welfare-maximizing path rather than on the Guo and Lansing saddle-path solution.

\footnotetext{
${ }^{13}$ A similar stabilization policy in which the tax would be redistributed in guise of a proportional lump sum transfer $T(t)=\tau(t) \bar{Y}(t)$ (where $\bar{Y}$ represents the average economy-wide levels of output) would make the agents better-off compared to the original Guo and Lansing policy. However, this policy does not change the main conclusions we draw in this section.

${ }^{14}$ In comparison, the original condition for indeterminacy and determinacy in Benhabib and Farmer [1994] and Guo and Lansing [1998] are respectively $\beta-1-\chi>0$ and $\beta(1-\phi)-1-\chi<0$.
} 


\subsection{Alternative economic policy}

Actually, the welfare-maximizing path can be selected through an alternative stabilization policy which is able to coordinate over time the agents on a given deterministic path.

Assume that the stationary equilibrium is indeterminate and that the government aims at coordinating the expectations on a deterministic indeterminate path characterized by the initial level of consumption and labor $\left(\bar{C}_{0}, \bar{L}_{0}\right)$. The expected rate of returns on capital is $\bar{r}_{0} \equiv a K_{0}^{\alpha-1} \bar{L}_{0}^{\beta}$. The economic policy consists in subsidizing or taxing production such that the rate of returns on capital equals $\bar{r}_{0}$ by fixing a tax rate $\tau_{0}$ (possibly negative) at the first period. Firms maximize their profit $\Pi_{0}$ :

$$
\Pi_{0}=\left(1-\tau_{0}\right) Y_{0}-r_{0} K_{0}-w_{0} L_{0},
$$

with:

$$
\tau_{0}=1-\bar{r}_{0} / r_{0}
$$

Since $K_{0}$ and $\bar{r}_{0}$ are predetermined, the equality of the after-tax rental rate of capital to the after-tax productivity of capital determines the quantity of labor at time 0 :

$$
L_{0}=\left(\bar{r}_{0} / a K_{0}^{\alpha}\right)^{1 / \beta}=\bar{L}_{0} .
$$

Simultaneously, the couple $\left(K_{0}, L_{0}\right)$ determines the equilibrium value of the first period after-tax real wage satisfying the second first order condition of profit maximization:

$$
w_{0}=\left(1-\tau_{0}\right) \frac{b Y_{0}}{L_{0}}
$$

Finally, the first order condition (respect to labor) determines consumption at time 0 , that is $\bar{C}_{0}$, which in turn determines the variation of capital and consumption at the next period via the law of motion of capital and the Euler equation. If the agents are rational, and we assume they are, they cannot switch to another equilibrium (which would be in contradiction with the FOCs) and are able to determine the triple $\left(K_{t}, L_{t}, C_{t}\right)$ at any time $t$. Of course, expectations can be coordinated by fixing the real wage or the rental rate of any period, not especially the first one.

\section{Conclusion}

In this paper, we have proposed a possible use of the linear-quadratic approximation already investigated by Benigno and Woodford [2006a, 2006b] in various economic problems: a welfare-ranking of alternative deterministic equilibrium paths in a one-sector growth model exhibiting local indeterminacy under a plausible degree of social increasing returns. The different equilibrium paths are ranked according to their initial value of consumption. We have shown that the initial value of consumption is all the higher and the welfare-maximizing path all the less monotonic since the degree of increasing returns is high. A direct implication of these findings is that progressive or countercyclical tax policies, as those developed by Guo and Lansing [1998], able to pin down expectations on a unique and smooth equilibrium path are likely to be welfare-diminishing in the presence of a sufficiently high productive externality compared to an indeterminate (deterministic or stochastic) equilibrium trajectory. Thus, the welfare-maximizing path provides an interesting second-best equilibrium when it is not possible to say anything about the nature of the social planer's trajectory.

At last, we have presented an alternative stabilization policy that pins down expectations on this welfare-maximizing path. In absence of precise knowledge of the optimal trajectory, this stabilization policy represents at least welfare improvement properties for economic agents compared to a laissez-faire or to a Guo and Lansing policy. 


\section{A Appendix}

\section{A.1 Dynamical system and steady state}

Let $H$ be the current value Hamiltonian of the programme:

$$
H(K, u, C, L, \Lambda)=\ln C-\frac{L^{1+\chi}}{1+\chi}+\Lambda\left(A[u K]^{a} L^{b}-\tau u^{\theta} K-C\right) .
$$

with $A=\left([u K]^{a} L^{b}\right)^{\gamma}$ and $Y=A[u K]^{a} L^{b}=[u K]^{\alpha} L^{\beta}$ (at the symmetric equilibrium).

The first-order conditions for an interior solution are:

$$
\begin{aligned}
& \frac{\partial H}{\partial u}(K, u, C, L, \Lambda)=0 \Longleftrightarrow \tau u^{\theta}=\frac{a}{\theta} \frac{Y}{K} \\
& \frac{\partial H}{\partial C}(K, u, C, L, \Lambda)=0 \Longleftrightarrow \Lambda=\frac{1}{C} \\
& \frac{\partial H}{\partial L}(K, u, C, L, \Lambda)=0 \Longleftrightarrow L^{\chi}=\Lambda b \frac{Y}{K} \\
& \frac{\partial H}{\partial K}(K, u, C, L, \Lambda)=-\dot{\Lambda}-\rho \Lambda \Longleftrightarrow \frac{\dot{\Lambda}}{\Lambda}=\rho+\tau u^{\theta}-\frac{a Y}{K} .
\end{aligned}
$$

The production function at the equilibrium can be rewritten in log-terms:

$$
y=\alpha k+\alpha v+\beta l
$$

where $x=\ln X$ and $v=\ln u$. Then, using equations (14) to (16) and substituting the value of $u, \Lambda$ and $L$ respect to $Y, C$ and $K$ in the production function, one gets the following linear relationship:

$$
y-k=\mu_{0}+\mu_{1} k+\mu_{2} c
$$

with $\mu_{0}=-\frac{\alpha(1+\chi) \ln (a / \theta \tau)+\theta \beta \ln b}{\theta \beta+\alpha(1+\chi)-\theta(1+\chi)}, \mu_{1}=\frac{\theta[(1-\alpha)(1+\chi)-\beta]}{\theta \beta+\alpha(1+\chi)-\theta(1+\chi)}$ and $\mu_{2}=\frac{\theta \beta}{\theta \beta+\alpha(1+\chi)-\theta(1+\chi)}$. And finally, the global dynamics of the model can be derived as the system of two equations in two unknowns, $c$ and $k$, expressed in equations (3) and (4).

The steady state equilibrium can then be derived by equalizing $\dot{C}$ and $\dot{K}$ to zero:

$$
\begin{aligned}
L_{s} & =\left(\frac{(\rho+\delta)(1-a)}{\rho+\delta(1-a)}\right)^{\frac{1}{1-\chi}} \\
K_{s} & =\left(\frac{a L_{s}^{\beta}}{\rho+\delta}\right)^{\frac{1}{1-\alpha}} \\
C_{s} & =\frac{(\rho+\delta(1-a))}{a}\left(\frac{a L_{s}^{\beta}}{\rho+\delta}\right)^{\frac{1}{1-\alpha}},
\end{aligned}
$$

where $\delta \equiv \tau u_{s}^{\theta}$ is the steady state value of the depreciation rate of capital. The value given to the parameter $\delta$ imposes the value of $\theta$ : at the steady state, $\delta=\frac{\rho}{\theta-1}$.

\section{A.2 Slopes of the stable arms}

The Jacobian matrix of the system formed by equation (3) and (4) is:

$$
J=\left(\begin{array}{cc}
{\left[1+\mu_{1}\left(1-\frac{a}{\theta}\right)\right] \Psi-\delta} & {\left[\mu_{2}\left(1-\frac{a}{\theta}\right)-1\right] \Psi+\delta} \\
a \mu_{1}\left(1-\frac{1}{\theta}\right) \Psi & a \mu_{2}\left(1-\frac{1}{\theta}\right) \Psi-\lambda_{i}
\end{array}\right) .
$$

Let $\xi_{i}=\left(v_{1 i}, v_{2 i}\right)^{T}, i=\{1,2\}$, the eigenvectors of the system defined such that:

$$
\left(\begin{array}{cc}
{\left[1+\mu_{1}\left(1-\frac{a}{\theta}\right)\right] \Psi-\delta-\lambda_{i}} & {\left[\mu_{2}\left(1-\frac{a}{\theta}\right)-1\right] \Psi+\delta} \\
a \mu_{1}\left(1-\frac{1}{\theta}\right) \Psi & a \mu_{2}\left(1-\frac{1}{\theta}\right) \Psi-\lambda_{i}
\end{array}\right)\left(\begin{array}{l}
v_{1 i} \\
v_{2 i}
\end{array}\right)=\left(\begin{array}{l}
0 \\
0
\end{array}\right) .
$$

The slope of the stable arm associated to $\xi_{i}$ at the stationary equilibrium is $v_{2 i} / v_{1 i}$. We want to show that:

$$
\frac{v_{22}}{v_{12}}>\frac{v_{21}}{v_{11}}>0
$$


or equivalently that the slope of the stable arm associated to $\xi_{2}$ is steeper than the slope of the stable arm associated to $\xi_{1}$ at the stationary equilibrium.

According to system (18), notice first that:

$$
\frac{v_{2 i}}{v_{1 i}}=\frac{\lambda_{i}+\delta-\left[1+\mu_{1}\left(1-\frac{a}{\theta}\right)\right] \Psi}{\left.\left[\mu_{2}\left(1-\frac{a}{\theta}\right)-1\right]\right) \Psi+\delta}=\frac{a \mu_{1}\left(1-\frac{1}{\theta}\right) \Psi}{\lambda_{i}-a \mu_{2}\left(1-\frac{1}{\theta}\right) \Psi} .
$$

Moreover when the condition for local indeterminacy is satisfied, that is when $\beta>\frac{\theta-\alpha}{\theta}(1+\chi)$, with $\theta>\alpha$, we have that $\mu_{2}>0$ and then the denominator of (19) is negative. On the other hand the sign of the numerator is negative when $\mu_{1}<0$, or equivalently $\beta>(1-\alpha)(1+\chi)$. This inequality is indeed always verified under the local indeterminacy condition since $\frac{\theta-\alpha}{\theta}>1-\alpha$ for any choice of the parameters. This proves that $\frac{v_{2 i}}{v_{1 i}}$ is always greater than zero.

Finally since $\lambda_{1}<\lambda_{2}$ it follows immediately from equation (19) that the slope of the stable arm associated to $\xi_{2}, v_{22} / v_{12}$, is steeper than the slope of the stable arm associated to $\xi_{1}, v_{21} / v_{11}$. If we assume the economy starts with an initial stock of capital lower (resp. greater) than its steady state value, $\tilde{k}(0)<0$ (resp. $\tilde{k}(0)>0$ ) and from equations $(9)$ and $(10)$ it is easily deduced that $c_{0, \xi_{1}}>c_{0, \xi_{2}}$ (resp. $\left.c_{0, \xi_{1}}<c_{0, \xi_{2}}\right)$.

\section{A.3 Monotonic paths}

Monotonicity of consumption paths occurs provided the equation $d \tilde{c}(t) / d t=0$ has no solution. This means that there is no $t \in \mathbb{R}^{+}$such that for any $\left(\eta_{1}, \eta_{2}\right)$ :

$$
\eta_{1} \lambda_{1} v_{21} e^{\lambda_{1} t}+\eta_{2} \lambda_{2} v_{22} e^{\lambda_{2} t}=0
$$

that is:

$$
t=\frac{1}{\lambda_{2}-\lambda_{1}} \ln \left(\frac{v_{22} \tilde{k}(0)-v_{12} \tilde{c}(0)}{v_{21} \tilde{k}(0)-v_{11} \tilde{c}(0)} \frac{\lambda_{1}}{\lambda_{2}} \frac{v_{21}}{v_{22}}\right)
$$

A solution exists if and only if $E \equiv \frac{v_{22} \tilde{k}(0)-v_{12} \tilde{c}(0)}{v_{21} \tilde{k}(0)-v_{11} \tilde{c}(0)}>0$. Define: $c(0) \equiv c_{0, \xi_{2}}+\varepsilon$. In this case relation $E$ becomes

$$
E=\frac{-v_{12}^{2} \varepsilon}{\left[v_{12} v_{21}-v_{11} v_{22}\right] \tilde{k}(0)-v_{11} v_{12} \varepsilon}
$$

where $v_{i j}<0$ for any $i, j=\{1,2\}$ (as shown in appendix $A .2$ ).

If $\varepsilon>0$, equation (20) as a solution if and only if $\left[v_{12} v_{21}-v_{11} v_{22}\right] \tilde{k}(0)-v_{11} v_{12} \varepsilon<0$, that is for:

$$
\begin{aligned}
\varepsilon & >\left[\frac{v_{21}}{v_{11}}-\frac{v_{22}}{v_{12}}\right] \tilde{k}(0) \\
& >c_{0, \xi_{1}}-c_{0, \xi_{2}},
\end{aligned}
$$

or equivalently for:

$$
c(0)>c_{0, \xi_{1}} \text {. }
$$

If $\varepsilon<0$, equation (20) as a solution if and only if $\left[v_{12} v_{21}-v_{11} v_{22}\right] \tilde{k}(0)-v_{11} v_{12} \varepsilon>0$, that is for:

$$
\begin{aligned}
\varepsilon & <\left[\frac{v_{22}}{v_{12}}-\frac{v_{21}}{v_{11}}\right] \tilde{k}(0) \\
& <c_{0, \xi_{2}}-c_{0, \xi_{1}},
\end{aligned}
$$

or equivalently for:

$$
c(0)<c_{0, \xi_{2}}-\varepsilon^{*},
$$

with $\varepsilon^{*} \equiv c_{0, \xi_{1}}-c_{0, \xi_{2}}$, which is positive (resp. negative) according to Appendix $A .2$ provided $K(0)<K_{s}$ (resp. $\left.K(0)>K_{s}\right)$.

Thus consumption paths have a monotonic behavior if and only if $c_{0} \in\left[c_{0, \xi_{2}}-\varepsilon^{*}, c_{0, \xi_{1}}\right]$ for $K(0)<K_{s}$ and $c_{0} \in\left[c_{0, \xi_{1}}, c_{0, \xi_{2}}-\varepsilon^{*}\right]$ for $K(0)>K_{s}$. 


\section{A.4 Solution of some limits}

The trace and determinant of the Jacobian matrix $J$ are the following:

$$
\begin{aligned}
\operatorname{Det}(J) & =a \Psi(\Psi-\delta)\left(1-\frac{1}{\theta}\right)\left(\mu_{1}+\mu_{2}\right) \\
\operatorname{Tr}(J) & =\left[1+\mu_{1}\left(1-\frac{a}{\theta}\right)+a \mu_{2}\left(1-\frac{1}{\theta}\right)\right] \Psi-\delta
\end{aligned}
$$

When the condition for indeterminacy holds, one can see immediately that $\operatorname{Tr}(J)$ tends to $-\infty$ and $\operatorname{Det}(J)$ tends to $+\infty$ as $\beta \frac{\theta}{\theta-\alpha}-1-\chi$ tends to zero. Moreover the two limits have the same "order" of convergence. Now consider the following limits:

$$
\lim _{\beta \frac{\theta}{\theta-\alpha} \rightarrow 1+\chi} \lambda_{1}=\lim _{\beta \frac{\theta}{\theta-\alpha} \rightarrow 1+\chi} \frac{\operatorname{Tr}(J)-|\operatorname{Tr}(J)| \sqrt{1-4 \frac{\operatorname{Det}(J)}{\operatorname{Tr}(J)^{2}}}}{2}=\lim _{\beta \frac{\theta}{\theta-\alpha} \rightarrow 1+\chi} \frac{\operatorname{Tr}(J)-|\operatorname{Tr}(J)|}{2}=-\infty
$$

Multiplying and dividing by $\operatorname{Tr}(J)-|\operatorname{Tr}(J)| \sqrt{1-4 \frac{\operatorname{Det}(J)}{\operatorname{Tr}(J)^{2}}}$, we get:

$$
\begin{aligned}
\lim _{\beta \frac{\theta}{\theta-\alpha} \rightarrow 1+\chi} \lambda_{2} & =\lim _{\beta \frac{\theta}{\theta-\alpha} \rightarrow 1+\chi} \frac{\operatorname{Tr}(J)+|\operatorname{Tr}(J)| \sqrt{1-4 \frac{\operatorname{Det}(J)}{\operatorname{Tr}(J)^{2}}}}{2} \\
& =\lim _{\beta \frac{\theta}{\theta-\alpha} \rightarrow 1+\chi} \frac{2 \operatorname{det} J}{\operatorname{tr} J-|\operatorname{tr} J| \sqrt{1-4 \frac{\operatorname{det} J}{(\operatorname{trJ})^{2}}}} \\
& =\lim _{\beta \frac{\theta}{\theta-\alpha} \rightarrow 1+\chi} \frac{\operatorname{Det}(J)}{\operatorname{Tr}(J)}=a(\Psi-\delta) \frac{1-\alpha}{a-\alpha}
\end{aligned}
$$




\section{References}

[1] Benigno, P. and Woodford, M. (2006a). "Optimal Taxation in an RBC Model: A Linear-Quadratic Approach". Journal of Economic Dynamics and Control 30, 1445-1489;

[2] Benigno, P. and Woodford, M. (2006b). "Linear-Quadratic Approximation of Optimal Policy Problems". NBER Working Paper, No. 12672.

[3] Benhabib, J. and Farmer, R. (1994). "Indeterminacy and increasing returns". Journal of Economic Theory 63, 19-41;

[4] Benhabib, J. and Farmer, R. (1996). "Indeterminacy and sector-specific externalities". Journal of Monetary Economics 37, 421-443;

[5] Christiano, L. and Harrison, S. (1999). "Chaos, sunspots and automatic stabilizers". Journal of Monetary Economics 44, 3-31;

[6] Federal Reserve Bank (2008). "Industrial Production and Capacity Utilization: The 2008 Annual Revision". Federal Reserve statistical release, G.17 (419) 2008 Historical and Annual Revision;

[7] Fleming, Wendell H. (1971). "Stochastic Control for Small Noise Intensities". SIAM Journal of Control, 9, 473-517;

[8] Guo, J. and Lansing, K. (1998). "Indeterminacy and stabilization policy". Journal of Economic Theory 82, 481-490;

[9] Guo, J.T. and Harrison, S. (2001). "Tax Policy and Stability in a Model with Sector-Specific Externalities". Review of Economic Dynamics 4, 75-89;

[10] Greenwood, Jeremy, Zvi Hercowitz, Gregory W. Huffman (1988). "Investment, Capacity Utilization, and the Real Business Cycle". American Economic Review, 78 (3), 402-417;

[11] Hansen, Gary D. (1985). "Indivisible Labor and the Business Cycle". Journal of Monetary Economics, 16, 309-325;

[12] Kim, J. and Kim, H.S. (2004). "Spurious Welfare Reversal in International Business Cycle Models". Journal of International Economics, 60, 471-500;

[13] Magill, Michael J.P. (1977). "A Local Analysis of N-Sector Capital Accumulation under Uncertainty". Journal of Economic Theory, 15, 211-218;

[14] Nadiri, M. Ishaq and Prucha Ingmar R. (1997). "Estimation of the Depreciation Rate of Physical and R\&D Capital in the U.S. Total Manufacturing Sector". NBER Working Paper, No. W4591.

[15] Russell, T. and Zecevic A. (1998). "Lyapunov stability, regions of attraction, and indeterminate growth paths". Economics Letters, 58 (3), 319-324; 
[16] Russell, T. and Zecevic A. (2000). "Indeterminate Growth Paths and Stability". Journal of Economic Dynamics and Control, 24, 39-62;

[17] Schmitt-Grohe, S. and Uribe, M. (2004). "Solving Dynamic General Equilibrium Models Using a Second-Order Approximation to the Policy Function". Journal of Economic Dynamics and Control $28,755-775$

[18] Sim, N.C.S. (2005). "Indeterminacy, Stabilization Policy and Returns to Scale: A Re-Investigation". Contributions to Macroeconomics 5, art.3; 


\section{Working Papers of the Center of Economic Research at ETH Zurich}

(PDF-files of the Working Papers can be downloaded at www.cer.ethz.ch/research).

08/99 M. Bambi and A. Saïdi

Increasing Returns to Scale and Welfare: Ranking the Multiple Deterministic Equilibria

08/98 M. Bambi

Unifying time-to-build theory

08/97 H. Gersbach and R. Winkler

International Emission Permit Markets with Refunding

08/96 K. Pittel and L. Bretschger

Sectoral Heterogeneity, Resource Depletion, and Directed Technical Change: Theory and Policy

08/95 M. D. König, S. Battiston, M. Napoletano and F. Schweitzer

The Efficiency and Evolution of R\&D Networks

08/94 H. Gersbach and F. Mühe

Vote-Buying and Growth

08/93 H. Gersbach

Banking with Contingent Contracts, Macroeconomic Risks, and Banking Crises

08/92 J. Daubanes

Optimal taxation of a monopolistic extractor: Are subsidies necessary?

08/91 R. Winkler

Optimal control of pollutants with delayed stock accumulation

08/90 S. Rausch and T. F. Rutherford

Computation of Equilibria in OLG Models with Many Heterogeneous Households

08/89 E. J. Balistreri, R. H. Hillberry and T. F. Rutherford

Structural Estimation and Solution of International TradeModels with Heterogeneous Firms

08/88 E. Mayer and O. Grimm

Countercyclical Taxation and Price Dispersion

08/87 L. Bretschger

Population growth and natural resource scarcity: long-run development under seemingly unfavourable conditions

08/86 M. J. Baker, C. N. Brunnschweiler, and E. H. Bulte

Did History Breed Inequality? Colonial Factor Endowments and Modern Income Distribution 
08/85 U. von Arx and A. Ziegler

The Effect of CSR on Stock Performance: New Evidence for the USA and Europe

08/84 H. Gersbach and V. Hahn

Forward Guidance for Monetary Policy: Is It Desirable?

08/83 I. A. MacKenzie

On the Sequential Choice of Tradable Permit Allocations

08/82 I. A. MacKenzie, N. Hanley and T. Kornienko

A Permit Allocation Contest for a Tradable Pollution Permit Market

08/81 D. Schiess and R. Wehrli

The Calm Before the Storm? - Anticipating the Arrival of General Purpose Technologies

08/80 D. S. Damianov and J. G. Becker

Auctions with Variable Supply: Uniform Price versus Discriminatory

08/79 H. Gersbach, M. T. Schneider and O. Schneller

On the Design of Basic-Research Policy

08/78 C. N. Brunnschweiler and E. H. Bulte

Natural Resources and Violent Conflict: Resource Abundance, Dependence and the Onset of Civil Wars

07/77 A. Schäfer, S. Valente

Habit Formation, Dynastic Altruism, and Population Dynamics

07/76 R. Winkler

Why do ICDPs fail? The relationship between subsistence farming, poaching and ecotourism in wildlife and habitat conservation

$07 / 75$ S. Valente

International Status Seeking, Trade, and Growth Leadership

07/74 J. Durieu, H. Haller, N. Querou and P. Solal

Ordinal Games

07/73 V. Hahn

Information Acquisition by Price-Setters and Monetary Policy

07/72 H. Gersbach and H. Haller

Hierarchical Trade and Endogenous Price Distortions

07/71 C. Heinzel and R. Winkler

The Role of Environmental and Technology Policies in the Transition to a Lowcarbon Energy Industry

07/70 T. Fahrenberger and H. Gersbach

Minority Voting and Long-term Decisions 
07/69 H. Gersbach and R. Winkler

On the Design of Global Refunding and Climate Change

07/68 S. Valente

Human Capital, Resource Constraints and Intergenerational Fairness

07/67 O. Grimm and S. Ried

Macroeconomic Policy in a Heterogeneous Monetary Union

07/66 O. Grimm

Fiscal Discipline and Stability under Currency Board Systems

07/65 M. T. Schneider

Knowledge Codification and Endogenous Growth

07/64 T. Fahrenberger and H. Gersbach

Legislative Process with Open Rules

07/63 U. von Arx and A. Schäfer

The Influence of Pension Funds on Corporate Governance

07/62 H. Gersbach

The Global Refunding System and Climate Change

06/61 C. N. Brunnschweiler and E. H. Bulte

The Resource Curse Revisited and Revised: A Tale of Paradoxes and Red Herrings

06/60 R. Winkler

Now or Never: Environmental Protection under Hyperbolic Discounting

06/59 U. Brandt-Pollmann, R. Winkler, S. Sager, U. Moslener and J.P. Schlöder Numerical Solution of Optimal Control Problems with Constant Control Delays

06/58 F. Mühe

Vote Buying and the Education of a Society

06/57 C. Bell and H. Gersbach

Growth and Enduring Epidemic Diseases

06/56 H. Gersbach and M. Müller

Elections, Contracts and Markets

06/55 S. Valente

Intergenerational Transfers, Lifetime Welfare and Resource Preservation

06/54 H. Fehr-Duda, M. Schürer and R. Schubert

What Determines the Shape of the Probability Weighting Function?

06/53 S. Valente

Trade, Envy and Growth: International Status Seeking in a Two-Country World

06/52 K. Pittel

A Kuznets Curve for Recycling 